\title{
A Facile Synthesis of (Z)-2-((5-(4-fluorobenzylidene)-4-oxo-4,5- dihydrothiazol-2-yl) amino) Substituted Acid Using Microwave Irradiation and Conventional Method
}

\author{
Dattatraya N. Pansare, Devanand B. Shinde*
}

Department of Chemical Technology, Babasaheb Ambedkar Marathwada University, Aurangabad 431 004, India.

\begin{abstract}
A new effective approach to the synthesis of some new (Z)-2-((5-(4-fluorobenzylidene)-4-oxo-4,5dihydrothiazol-2-yl)amino) substituted acid 6a-1 is reported under microwave irradiation as well as conventional conditions.
\end{abstract}

Keywords: Amino acids, Knoevenagel condensation, Microwave irradiation, Rhodanine.

\section{INTRODUCTION}

The structural and therapeutic diversity coupled with commercial viability of small heterocyclic molecules have fascinated organic and medicinal chemists. In recent years, 4-thiazolidinones (Rhodanine) have been extensively investigated as class of compounds. Rhodanine have many interesting activity profiles namely COX-1 inhibitors [1] inhibitors of the bacterial enzyme MurB [2]. The rhodanine scaffold is a central part of biologically active compounds with various applications and uses [3, 4] such as anti-microbial $[5,6]$ anti-malarial [7] anti-HIV agents [8 - 11] antiinflammatory [12, 13] anti-fungal [14] anti-cancer [15] antidiabetic [16] and antitubercular [17, 18]. For the discovery of new lead structures in drug discovery, based on high throughput screening, synthetic methodologies are required which deliver highly diverse derivatives in a timely manner. Under these circumstances, microwave-assisted chemistry appears to be a promising synthetic method [19]. Utility of microwave irradiation [20] (MW) to carry out organic reaction has now become a regular feature. The main benefits of performing the reaction under microwave conditions are the significant rate enhancements and the higher product yields in minimum time requirement. Here, we wish to mention the development and implementation of a methodology, allowing for the synthesis of some new (Z)-2- ((5-(4-fluorobenzylidene)

\footnotetext{
* Address correspondence to this author at the Department of Chemical Technology, Babasaheb Ambedkar Marathwada University, Aurangabad 431 004, India; Tel No.:+91 0240-2403308; Fax No.: +91 0240-2400413; E-mail: dbsdattatraya10@rediffmail.com
}

-4-oxo-4,5-dihydrothiazol- 2-yl)amino) substituted acid (6a-l) derivatives. The rhodanine has been known for over 50 years, so there have been several attempts to design antimicrobial agents based on this heterocycle. There are various reports available on rhodanine derivatives as antimicrobial agents [21 - 25]. These reports suggested that a chain containing free carboxyl group at rhodanine nucleus was important to the observed as levels of antimicrobial activity $[26,27]$. The reported reactions under microwave irradiation as well as conventional method proceed in short reaction times and are good to excellent yields. With this in mind, we initiated a program by using microwave irradiation and conventional method to synthesized rhodanine derivatives, having amino acids chain as antimicrobial agent by preparing hybrid molecules having the similar features of reported potent antimicrobial agents (Fig. 1).

In continuation of our work [28], on the synthesis of bioactive compounds, we have synthesized some rhodanine analogues. The synthetic protocols employed for the synthesis of rhodanine derivatives $\mathbf{3}$ and $\mathbf{4}$ are presented in Scheme 1.

\section{RESULT AND DISCUSSION}

The first part of the study was aimed at optimizing the reaction conditions. The screening of model reaction of (Z)-2-((5- (4-fluorobenzylidene) -4-oxo-4,5dihydrothiazol-2 -yl)amino)propanoic acid 6a (Scheme 2, Table. 1). We have developed the protocol for the synthesis of compound $\mathbf{6 a}$ by condensation of compounds 4 and 5a. After the initial success in ethanol, we screened various solvents, bases, time and yield; the results are shown in Table. 1. The reaction of compound $4(1 \mathrm{mmol})$ and compound $\mathbf{5 a}(1.2 \mathrm{mmol})$, catalyzed by 
various bases and various solvents were selected as a model reaction to optimize the reactive conditions. In terms of the effect of solvents and bases on the condensation reaction, potassium carbonate was found to be the better base and ethanol was found to be the best solvent for the reaction (Table. 1, entry 11); other solvents, including dichloromethane (DCM), acetic acid, methanol and toluene were less efficient (Table. 1, entries 2-5, 7-10 and 12-15). Nevertheless, all of these yields were generally low before further optimizations. Ethanol gave the corresponding product in a $50-90 \%$ yield, which was the best among these solvents (Table. 1, entries 1, 6 and 11). To increase the efficiency of the condensation reaction, the effects of different bases were investigated (Table. 1, entries 1-15). Potassium carbonate exhibited the best performance with used solvents and gave better yield, (Table. 1, entries 11-15). Diethylamine and triethylamine gave lower yields with other solvents, but gave better yield in combination with ethanol as a solvent (Table. 1, entries 1 and 6). All the reactions were carried out in equimolar amounts of each compound in $1 \mathrm{~mL}$ of solvent. Among these reactions same amounts of the solvent, namely 1 $\mathrm{mL}$ of ethanol turned out to be the best choice with yields of $50 \%, 65 \%$ and $90 \%$ (Table. 1, entries 1, 6 and 11). We would like to mention here that ethanol as a solvent with potassium carbonate as base was the best choice with a yield of $90 \%$ and less time required for the completion of the reaction (Table. 1, entry 11). Thus we decided to carry out the reactions in ethanol with potassium carbonate. Simultaneously, we also performed microwave-assisted reactions. In addition the reaction time for microwave-assisted reactions was much shorter than the same reactions in all of our studied substrates. As a result the reaction time was shortened, thermal decomposition also minimized, resulting in higher isolated yields (Table. 2).

Table 1. Screening of bases, solvents, reaction time and yield for the synthesis (6a) ${ }^{\mathrm{a}}$.

\begin{tabular}{|c|c|c|c|c|}
\hline Entry & Base & Solvent & Time (min) & Yield $^{\mathrm{b}}(\%)$ \\
\hline 1 & Diethylamine & Ethanol & 120 & 50 \\
\hline 2 & Diethylamine & Methanol & 130 & 30 \\
\hline 3 & Diethylamine & Acetic acid & 150 & 35 \\
\hline 4 & Diethylamine & DCM & 110 & 40 \\
\hline 5 & Diethylamine & Toluene & 140 & 30 \\
\hline 6 & Triethylamine & Ethanol & 100 & 65 \\
\hline 7 & Triethylamine & Methanol & 110 & 40 \\
\hline 8 & Triethylamine & Acetic acid & 130 & 30 \\
\hline 9 & Triethylamine & DCM & 120 & 45 \\
\hline 10 & Triethylamine & Toluene & 125 & 35 \\
\hline 11 & Potassium carbonate & Ethanol & 30 & 90 \\
\hline 12 & Potassium carbonate & Methanol & 40 & 80 \\
\hline 13 & Potassium carbonate & Acetic acid & 60 & 80 \\
\hline 14 & Potassium carbonate & DCM & 50 & 85 \\
\hline 15 & Potassium carbonate & Toluene & 70 & 75 \\
\hline
\end{tabular}

${ }^{a}$ All the reaction was carried out in 1:1.2 molar amounts of each compound in $1 \mathrm{~mL}$ of solvent.

${ }^{\mathrm{b}}$ Isolated yield.<smiles>O=Cc1ccc(F)cc1</smiles>

1<smiles>O=C1CSC(=S)N1</smiles>

2<smiles>O=C1NC(=S)S/C1=C\c1ccc(F)cc1</smiles>

3<smiles>CSC1=NC(=O)/C(=C/c2ccc(F)cc2)S1</smiles>

4

Scheme. 1. Synthesis of (Z)-5-(4-fluorobenzylidene)-2-(methylthio)thiazol-4(5H)-one (4). 
<smiles>CSC1=NC(=O)/C(=C/c2ccc(F)cc2)S1</smiles>

4<smiles>CC(N)C(=O)O</smiles>

$5 \mathbf{a}$<smiles>CC(NC1=NC(=O)/C(=C/c2ccc(F)cc2)S1)C(=O)O</smiles>

$6 a$

${ }^{\text {a }}$ Reaction condition: compound (4)(1 mmol), L-Alanine(5a)(1.2 mmol), base(1 mmol), solvent 1mL, rt. 30-150 min.

Scheme. 2. Screening of model reaction (Z)-2-((5-(4-fluorobenzylidene)-4-oxo-4,5-dihydrothiazol-2-yl)amino)propanoic acid (6a)

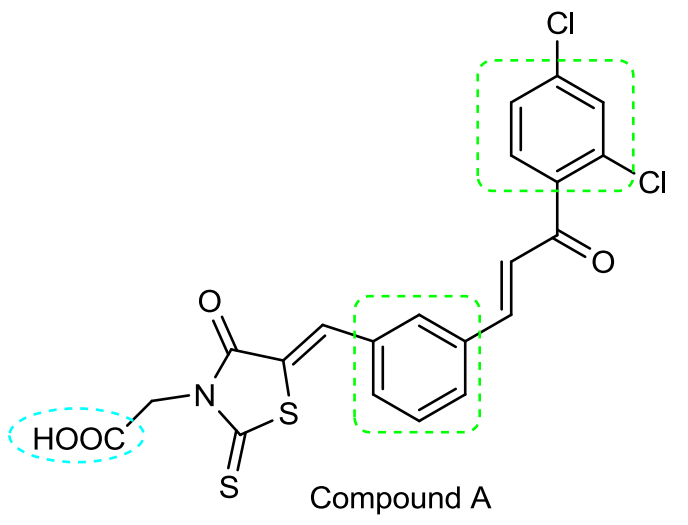

$(\mathrm{MIC}=1 \mu \mathrm{g} / \mathrm{mL})$

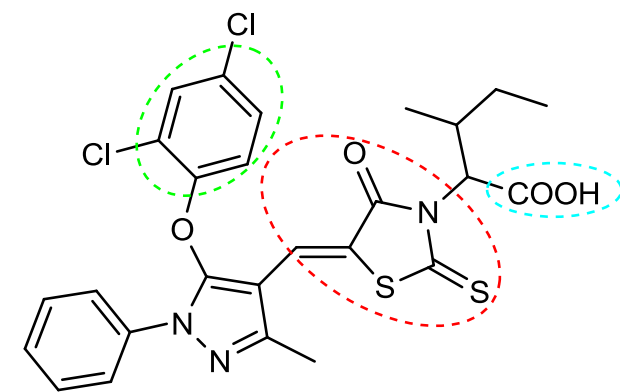

Compound B

$(\mathrm{MIC}=4 \mu \mathrm{g} / \mathrm{mL})$<smiles>O=C1NC(=S)S/C1=C\c1ccc(F)cc1</smiles>

3<smiles>CSC1=NC(=O)/C(=C/c2ccc(F)cc2)S1</smiles>

4<smiles>[R]C(NC1=NC(=O)/C(=C/c2ccc(F)cc2)S1)C(=O)O</smiles>

$6 a-1$

Fig. (1). Previously reported antibacterial agents and synthesized compounds.

The synthesized rhodanine derivatives by using microwave irradiation and conventional method 6a-l (Scheme 3, Table. 2). The compound 5-(4fluorobenzylidene)-2-thioxothiazolidin- 4-one $\mathbf{3}$ was prepared via a Knoevenagel condensation between 4fluorobenzaldehyde $\mathbf{1}$ and rhodanine $\mathbf{2}$. The compound 5-(4-fluorobenzylidene)-2-(methylthio)thiazol-4(5H)one 4 was obtained via reaction of 5-(4fluorobenzylidene)-2-thioxothiazolidin-4-one (3) with iodomethane in dichloromethane using triethylamine as catalyst [29]. Further, to expand the series, 2-((5-(4fluorobenzylidene)- 4-oxo-4, 5-dihydrothiazol -2- yl)amino) acid derivatives 6a-1 were synthesized reacting 5-(4-fluorobenzylidene)-2-(methylthio)thiazol-4(5H)one 4 with various amino acids $\mathbf{5 a - 1}$ in ethanol using $\mathrm{K}_{2} \mathrm{CO}_{3}$ as catalyst [30].

In this reaction, there was displacement of a methyl sulfinyl group by amino acids from the $\mathrm{C} 2$ position of the thiazolone ring.

Rhodanine based compounds were synthesized by microwave irradiation (MW) as well as conventional heating with potassium carbonate and ethanol. 
Table 2. Physical data for synthesized rhodanine derivatives $6(\mathrm{a}-\mathrm{l})^{\mathrm{a}}$.

\begin{tabular}{|c|c|c|c|c|c|c|}
\hline \multirow{2}{*}{ Sr. No. } & \multirow{2}{*}{ Substituent (R) } & \multicolumn{2}{|c|}{ Time (min) } & \multicolumn{2}{|c|}{ Yield $^{\mathrm{b}}(\%)$} & \multirow{2}{*}{$\begin{array}{c}\text { Melting } \\
\text { point }\left({ }^{\circ} \mathbf{C}\right)\end{array}$} \\
\hline & & $\mathbf{M W}^{\mathbf{c}}$ & $\operatorname{Con}^{\mathrm{d}}$ & $\mathbf{M W}^{\mathrm{e}}$ & $\operatorname{Con}^{\mathrm{d}}$ & \\
\hline $6 a$ & -CH3 & 4 & 30 & 96 & 90 & $230-232$ \\
\hline $6 b$ & $-\mathrm{CH}(\mathrm{CH} 3) 2$ & 4 & 40 & 96 & 80 & $202-204$ \\
\hline $6 \mathrm{c}$ & $-\mathrm{CH}(\mathrm{CH} 3) \mathrm{CH} 2 \mathrm{CH} 3$ & 3 & 35 & 94 & 82 & $140-142$ \\
\hline $6 \mathrm{~d}$ & $-\mathrm{CH} 2 \mathrm{C} 6 \mathrm{H} 5$ & 3 & 45 & 93 & 80 & $170-172$ \\
\hline $6 e$ & $-\mathrm{CH} 2 \mathrm{CH} 2 \mathrm{SCH} 3$ & 3 & 45 & 94 & 82 & $156-158$ \\
\hline $6 f$ & $-\mathrm{CH} 2 \mathrm{CH}(\mathrm{CH} 3) 2$ & 4 & 35 & 94 & 84 & $233-235$ \\
\hline $6 g$ & $-\mathrm{CH} 2 \mathrm{OH}$ & 3 & 30 & 95 & 82 & $201-203$ \\
\hline $6 \mathrm{~h}$ & $-\mathrm{CH} 2 \mathrm{SH}$ & 4 & 35 & 94 & 84 & $206-208$ \\
\hline $6 \mathrm{i}$ & $-\mathrm{CH} 2 \mathrm{COOH}$ & 4 & 50 & 96 & 80 & $182-184$ \\
\hline $6 j$ & $1 \mathrm{NH}$ & 3 & 50 & 92 & 76 & 192-194 \\
\hline $6 \mathrm{k}$ & - $\mathrm{CH} 2 \mathrm{C} 6 \mathrm{H} 4 \mathrm{OH}$ & 3 & 45 & 92 & 72 & $150-152$ \\
\hline 61 & - $\mathrm{CHOHCH} 3$ & 3 & 35 & 94 & 78 & $170-172$ \\
\hline
\end{tabular}

${ }^{\mathrm{a}}$ Reaction condition (6a-l). Compound (4) (1 mmol), amino acids (5a-l) (1.2 mmol),

Method A: Microwave-assisted synthesis: potassium carbonate, ethanol, $70{ }^{\circ} \mathrm{C}, 3-4 \mathrm{~min}$.

Method B: Conventional synthesis: potassium carbonate, ethanol room temperature, 30-50 min.

${ }^{\mathrm{b}}$ Isolated yields.

${ }^{\mathrm{c}}$ Microwave-assisted.

${ }^{\mathrm{d}}$ Conventional condition.<smiles>CSC1=NC(=O)/C(=C/c2ccc(F)cc2)S1</smiles>

4<smiles>[R]C(N)C(=O)O</smiles>

5a-I

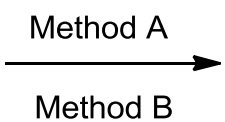

Method B<smiles>[R]C(NC1=NC(=O)/C(=C/c2ccc(F)cc2)S1)C(=O)O</smiles>

6a-I

$$
\begin{array}{rlrl}
R=5 a & =6 a=-\mathrm{CH}_{3} & & 5 e=6 e=-\mathrm{CH}_{2} \mathrm{CH}_{2} \mathrm{SCH}_{3} \\
5 b & =6 b=-\mathrm{CH}\left(\mathrm{CH}_{3}\right)_{2} & 5 f=6 f=-\mathrm{CH}_{2} \mathrm{CH}\left(\mathrm{CH}_{3}\right)_{2} \\
5 c=6 c=-C C_{3}\left(\mathrm{CH}_{3}\right) \mathrm{CH}_{2} \mathrm{CH}_{3} & 5 \mathrm{~g}=6 \mathrm{~g}=-\mathrm{CH}_{2} \mathrm{OH} \\
5 d=6 d=-\mathrm{CH}_{2} \mathrm{C}_{6} \mathrm{H}_{5} & 5 \mathrm{~h}=6 \mathrm{~h}=-\mathrm{CH}_{2} \mathrm{SH}
\end{array}
$$$$
5 i=6 i=-\mathrm{CH}_{2} \mathrm{COOH}
$$$$
5 \mathrm{j}=6 \mathrm{j}=-\mathrm{NH}_{\mathrm{NH}}^{\mathrm{N}}
$$$$
5 \mathrm{k}=6 \mathrm{k}=-\mathrm{CH}_{2} \mathrm{C}_{6} \mathrm{H}_{4} \mathrm{OH}
$$$$
5 \mathrm{I}=6 \mathrm{I}=-\mathrm{CHOHCH}_{3}
$$

${ }^{\mathrm{a}}$ Reaction condition:

Method A: Microwave-assisted synthesis: potassium carbonate, ethanol, $70{ }^{\circ} \mathrm{C}, 3-4 \mathrm{~min}$.

Method B: Conventional synthesis: potassium carbonate, ethanol room temperature, 30-50 min.

Scheme. 3. Synthesis of (Z)-2-((5-(4-fluorobenzylidene)-4-oxo-4,5-dihydrothiazol-2-yl) amino) substituted acid (6a-l) ${ }^{\mathrm{a}}$.

The physical data of the synthesized compounds are presented in (Table. 2). All the reactions proceeded well in 3-4 min. in microwave irradiation to give products in very good yields $(92-96 \%)$ and $30-50 \mathrm{~min}$ at conventional method to give products in very good yields $(72-90 \%)$. The purity of the synthesized compounds was checked by TLC and melting points were recorded on SRS Optimelt, melting point apparatus 
and are incorrected. The structure of the synthesized compounds was confirmed by IR, ${ }^{1} \mathrm{H}$ NMR, ${ }^{13} \mathrm{C}$ NMR and Mass spectral analysis.

\section{EXPERIMENTAL}

General Procedure for the Synthesis of Compounds (3)

In a $100 \mathrm{ml}$ round bottom flask, equimolar amounts of 4-fluorobenzaldehyde 1 ( $1 \mathrm{mmol})$, 2-thioxothiazolidin -4-one 2 ( $1 \mathrm{mmol})$, anhydrous sodium acetate $(1 \mathrm{mmol})$ were added in glacial acetic acid $(1 \mathrm{~mL})$. The mixture was stirred under reflux condition for $7 \mathrm{~h}$. The progress of reaction was monitored by TLC ( $20 \%$ ethyl acetate: $n-$ hexane). After completion of the reaction, the reaction mixture was poured into the icecold water. The precipitate was filtered off and washed with water $(3 \times 15$ $\mathrm{mL})$, dried and purified by recrystallization in ethanol as solvent to give $85 \%$ yield.

\section{(Z)-5-(4-fluorobenzylidene)-2-thioxothiazolidin-4-one (3)}

Yellow solid. Yield: $85 \%$. mp 226-228 ${ }^{\circ} \mathrm{C}$; ES-MS $\mathrm{m} / \mathrm{z}:$ 239. IR $v \max / \mathrm{cm}^{-1}$ : $3034(\mathrm{NH}), 2837(\mathrm{CH}-\mathrm{Ar})$, $1687(\mathrm{C}=\mathrm{O}), 1574(\mathrm{C}=\mathrm{C}), 1444(\mathrm{C}=\mathrm{N}), 1229(\mathrm{C}=\mathrm{S})$, $1069(\mathrm{C}-\mathrm{N}) .{ }^{1} \mathrm{H}$ NMR: äppm $=6.90-7.10(\mathrm{~d}, 2 \mathrm{H}, \mathrm{Ar}-\mathrm{H})$, 7.50-7.60 (d, 2H, Ar-H), $8.10(\mathrm{~s}, 1 \mathrm{H},=\mathrm{CH}), 7.90(\mathrm{~s}, 1 \mathrm{H}$, $\mathrm{NH}) .{ }^{13} \mathrm{C}$ NMR: $\delta \mathrm{ppm}=115.3,116.3,130.4,130.8$, $143.4,162.3,168.3,193.6$.

\section{General Procedure for the Synthesis of Compounds} (4)

In a $100 \mathrm{~mL}$ round bottom flask, the compound 3 (1 $\mathrm{mmol})$, iodomethane $(1.2 \mathrm{mmol})$ triethylamine $(1.2$ $\mathrm{mmol})$ were added to dichloromethane $(1 \mathrm{~mL})$ at room temperature, stirred reaction mixture for $1 \mathrm{~h}$ at room temperature. The progress of the reaction was monitored by TLC ( $10 \%$ chloroform: methanol). After completion of reaction the reaction mixture was concentrated in vacuo. The residue was washed with water $(3 \times 15 \mathrm{~mL})$ to afford the crude product. The crude product was recrystallized using ethanol as solvent to give yield in the range $75 \%$.

\section{(Z)-5-(4-fluorobenzylidene)-2-(methylthio)thiazol- 4(5H)-one (4)}

Yellow solid. Yield: $75 \%$. mp $143-145{ }^{\circ} \mathrm{C}$; ES-MS $\mathrm{m} / \mathrm{z}$ : 253. IR vmax $/ \mathrm{cm}^{-1}: 3015(\mathrm{CH}-\mathrm{Ar}), 1700(\mathrm{C}=\mathrm{O})$, $1594(\mathrm{C}=\mathrm{C}), 1483(\mathrm{C}=\mathrm{N}), 1153(\mathrm{C}-\mathrm{S}), 824(\mathrm{C}-\mathrm{N}) .{ }^{1} \mathrm{H}$ NMR: äppm $=2.80\left(\mathrm{~s}, 3 \mathrm{H}, \mathrm{SCH}_{3}\right), 7.50-7.75(\mathrm{~m}, 4 \mathrm{H}$, $\mathrm{Ar}-\mathrm{H}), 7.90(\mathrm{~s}, 1 \mathrm{H},=\mathrm{CH}) .{ }^{13} \mathrm{C} \mathrm{NMR}: \delta \mathrm{ppm}=14.2$, 115.4, 130.4, 132.3, 132.4, 133.5, 152.2, 162.1, 167.2.

\section{General Procedure for the Synthesis of Compounds (6a-l)}

\section{Method A: Microwave-Assisted Synthesis}

In a $100 \mathrm{ml}$ round bottom flask, the compound 4 (1 mmol), amino acids 5a-l (1.2 $\mathrm{mmol})$, potassium carbonates $(1.2 \mathrm{mmol})$, were added to ethanol $(1 \mathrm{~mL})$ was added and this mixture subjected to MW irradiation $(800 \mathrm{~W})$, at $70{ }^{\circ} \mathrm{C}$ temperature for 3-4 $\mathrm{min}$. The progress of reaction was monitored by TLC (10\% chloroform: methanol). After completion of reaction, the reaction mixture was concentrated in vacuo. The resulting solid was extracted with ethyl acetate for column chromatography. The column chromatography was performed using silica gel (200-300 mesh), eluted with ethyl acetate and petroleum ether $(1: 1, \mathrm{v} / \mathrm{v})$ to give product 6a-I (Yield: 92-96 \%).

\section{Method B: Conventional Synthesis}

In a $100 \mathrm{ml}$ round bottom flask, the compound 4 (1 mmol), amino acids 5a-l (1.2 $\mathrm{mmol})$, potassium carbonates $(1.2 \mathrm{mmol})$, were added to ethanol $(1 \mathrm{~mL})$ stirring 30-50 $\mathrm{min}$. at room temperature. The progress of the reaction was monitored by TLC $(10 \%$ chloroform: methanol). After completion of reaction, the reaction mixture was concentrated in vacuo. The resulting solid was extracted with ethyl acetate for column chromatography. Column chromatography was performed using silica gel (200-300 mesh), eluted with ethyl acetate and petroleum ether $(1: 1, \mathrm{v} / \mathrm{v})$ to give product 6a-1 (Yield: 72-90\%).

\section{(Z)-2-((5-(4-fluorobenzylidene)-4-oxo-4,5- dihydrothiazol-2-yl)amino)propanoic acid (6a)}

Yellow solid. Yield: 96\%. mp 230-232 ${ }^{\circ} \mathrm{C}$; ES-MS $\mathrm{m} / \mathrm{z}$ : 294. IR vmax/ $/ \mathrm{cm}^{-1}$ : $3342(\mathrm{OH}), 2966(\mathrm{CH}-\mathrm{Ar})$, $1723(\mathrm{C}=\mathrm{O}), 1593(\mathrm{C}=\mathrm{C}), 1504(\mathrm{C}=\mathrm{N}), 1158(\mathrm{C}-\mathrm{S}), 893$ $(\mathrm{C}-\mathrm{N}) .{ }^{1} \mathrm{H}$ NMR: äppm $=1.40-1.45\left(\mathrm{~d}, 3 \mathrm{H}, \mathrm{CH}_{3}\right)$, 4.60-4.80 (q, 1H, CH), 7.36-7.65 (m, 4H, Ar-H), 7.86 $(\mathrm{s}, 1 \mathrm{H},=\mathrm{CH}), 10.20(\mathrm{~s}, 1 \mathrm{H}, \mathrm{NH}), 12.65(\mathrm{~s}, 1 \mathrm{H}, \mathrm{OH}) .{ }^{13} \mathrm{C}$ NMR: $\delta p p m=16.9,53.7,115.4,130.3,130.7,132.6$, $152.2,158.3,167.2,174.7$.

\section{(Z)-2-((5-(4-fluorobenzylidene)-4-oxo-4,5- dihydrothiazol-2-yl)amino)-3-methylbutanoic acid (6b)}

Yellow solid. Yield: 96\%. mp 202-204 ${ }^{\circ} \mathrm{C}$; ES-MS $\mathrm{m} / \mathrm{z}: 322$. IR $v \max / \mathrm{cm}^{-1}$ : $3366(\mathrm{OH}), 3175(\mathrm{NH}), 2997$ $(\mathrm{CH}-\mathrm{Ar}), 1724(\mathrm{C}=\mathrm{O}), 1677(\mathrm{C}=\mathrm{C}), 1505(\mathrm{C}=\mathrm{N})$, 1235(C-S), $1160(\mathrm{C}-\mathrm{N}) .{ }^{1} \mathrm{H}$ NMR: äppm $=0.90-1.10(\mathrm{~d}$, $\left.6 \mathrm{H}, \mathrm{CH}_{3}\right), 2.11-2.25(\mathrm{~m}, 1 \mathrm{H}, \mathrm{CH}), 4.47-4.55(\mathrm{~d}, 1 \mathrm{H}$, $\mathrm{CH}), 7.35-7.65(\mathrm{~m}, 4 \mathrm{H}, \mathrm{Ar}-\mathrm{H}), 7.80(\mathrm{~s}, 1 \mathrm{H},=\mathrm{CH}), 9.90$ $(\mathrm{s}, 1 \mathrm{H}, \mathrm{NH}), 13.00(\mathrm{~s}, 1 \mathrm{H}, \mathrm{OH}) .{ }^{13} \mathrm{C} \mathrm{NMR}: \delta \mathrm{ppm}=18.9$, 
$30.2,61.3,115.3,130.4,130.8,132.6,152.2,158.3$ $162.2,167.2,174.3$.

\section{(Z)-2-((5-(4-fluorobenzylidene)-4-oxo-4,5-} dihydrothiazol-2-yl)amino)-3-methylpentanoic acid (6c)

Yellow solid. Yield: 94\%. mp 140-142 ${ }^{\circ} \mathrm{C}$; ES-MS $\mathrm{m} / \mathrm{z}$ : 336. IR $v \max / \mathrm{cm}^{-1}: 3523(\mathrm{OH}), 3180(\mathrm{NH}), 2964$ $(\mathrm{CH}-\mathrm{Ar}), 1703(\mathrm{C}=\mathrm{O}), 1554(\mathrm{C}=\mathrm{C}), 1503(\mathrm{C}=\mathrm{N}), 1228$ (C-S), $1158(\mathrm{C}-\mathrm{N}) .{ }^{1} \mathrm{H}$ NMR: äppm $=1.01-1.15(\mathrm{t}, 3 \mathrm{H}$, $\left.\mathrm{CH}_{3}\right), 1.23-1.31$ (d, 3H, $\left.\mathrm{CH}_{3}\right), 1.83-2.03\left(\mathrm{~m}, 3 \mathrm{H}, \mathrm{CH}_{2}\right.$, $\mathrm{CH}), 3.45-3.55$ (d, 1H, CH), 7.45-7.65 (m, 4H, Ar-H), $7.80(\mathrm{~s}, 1 \mathrm{H},=\mathrm{CH}), 10.15(\mathrm{~s}, 1 \mathrm{H}, \mathrm{NH}), 12.80$ (s, 1H, OH). ${ }^{13} \mathrm{C}$ NMR: $\delta p p m=11.2,15.3,25.2,37.3,58.6,115.3$, $130.2,130.8,132.6,152.2,158.3,162.2,167.3,174.6$.

\section{CONCLUSION}

In conclusion, we have successfully developed an easy access to a new series of 2-((5-(4fluorobenzylidene) -4-oxo-4, 5-dihydrothiazol -2yl)amino) acid derivatives 6a-l. The mild reaction conditions, good to excellent yields, easy workup, and easily available substrates make the reactions attractive for the preparation of compounds 6a-l. Efforts towards the synthesis of other important drug molecules with a rhodanine moiety by microwave irradiation as well as conventional method are ongoing in our laboratory. Besides, the technique has the advantage of being simple and allows the synthesis of rhodanine moiety in minimum time.

\section{ACKNOWLEDGEMENTS}

The authors are thankful to the Head, Department of Chemical Technology, Dr. Babasaheb Ambedkar Marathwada University, Aurangabad 431004 (MS) India, for providing the laboratory facility.

\section{REFERENCES}

[1] Look, G.C.; Schullek, J.R.; Homes, C.P.; Chinn, J.P.; Gordon, E.M.; Gallop, M.A. The identification of cyclooxygenase-1 inhibitors from 4-thiazolidinone combinatorial libraries. Bioorg. Med. Chem. Lett., 1996, 6, 707-712.

[http://dx.doi.org/10.1016/0960-894X(96)00097-2]

[2] Andres, C.J.; Bronson, J.J.; D’ Andrea, S.V.; Deshpande, M.S.; Falk, P.J.; Grant-Young, K.A.; Harte, W.E.; Ho, H.T.; Misco, P.F.; Robertson, J.G.; Stock, D.; Sun, Y.; Walsh, A.W. 4Thiazolidinones: novel inhibitors of the bacterial enzyme MurB. Bioorg. Med. Chem. Lett., 2000, 10(8), 715-717. [http://dx.doi.org/10.1016/S0960-894X(00)00073-1] [PMID: 10782671]

[3] Singh, S.P.; Parmar, S.S.; Raman, K.; Stenberg, V.I. Chemistry and biological activity of thiazolidinones. Chem. Rev., 1981, 81, 175-203.

[http://dx.doi.org/10.1021/cr00042a003]

[4] Brown, F.C. 4-Thiazolidinones. Chem. Rev., 1961, 61, 463-521.

[http://dx.doi.org/10.1021/cr60213a002]
[5] Kavitha, C.V.; Basappa, B.; Swamy, S.N.; Mantelingu, K.; Doreswamy, S.; Sridhar, M.A.; Shashidhara Prasad, J.; Rangappa, K.S. Synthesis of new bioactive venlafaxine analogs: novel thiazolidin-4-ones as antimicrobials. Bioorg. Med. Chem., 2006, 14(7), 2290-2299.

[http://dx.doi.org/10.1016/j.bmc.2005.11.017]

[PMID: 16338140]

[6] Shah, T.J.; Desai, V.A. Synthesis of some novel fluorinated 4thiazolidinones containing amide linkages and their antimicrobial screening. ARKIVOC, 2007, 14, 218-228.

[7] Solomon, V.R.; Haq, W.; Srivastava, K.; Puri, S.K.; Katti, S.B. Synthesis and antimalarial activity of side chain modified 4aminoquinoline derivatives. J. Med. Chem., 2007, 50(2), 394-398.

[http://dx.doi.org/10.1021/jm061002i] [PMID: 17228883]

[8] Rawal, R.K.; Tripathi, R.; Katti, S.B.; Pannecouque, C.; De Clercq, E. Design and synthesis of 2-(2,6-dibromophenyl)-3heteroaryl-1,3-thiazolidin-4-ones as anti-HIV agents. Eur. J. Med. Chem., 2008, 43(12), 2800-2806.

[http://dx.doi.org/10.1016/j.ejmech.2007.12.015] [PMID: 18242784]

[9] Balzarini, J.; Orzeszko, B.; Maurin, J.K.; Orzeszko, A. Synthesis and anti-HIV studies of 2-adamantyl-substituted thiazolidin-4-ones. Eur. J. Med. Chem., 2007, 42(7), 993-1003. [http://dx.doi.org/10.1016/j.ejmech.2007.01.003] [PMID: 17321639]

[10] Murugesan, V.; Tiwari, V.S.; Saxena, R.; Tripathi, R.; Paranjape, R.; Kulkarni, S.; Makwana, N.; Suryawanshi, R.; Katti, S.B. Lead optimization at C-2 and N-3 positions of thiazolidin-4-ones as HIV-1 non-nucleoside reverse transcriptase inhibitors. Bioorg. Med. Chem., 2011, 19(22), 6919-6926.

[http://dx.doi.org/10.1016/j.bmc.2011.09.018] [PMID: 21982685]

[11] Rawal, R.K.; Tripathi, R.; Katti, S.B.; Pannecouque, C.; De Clercq, E. Synthesis and evaluation of 2-(2,6-dihalophenyl)-3pyrimidinyl-1,3-thiazolidin-4-one analogues as anti-HIV-1 agents. Bioorg. Med. Chem., 2007, 15(9), 3134-3142.

[http://dx.doi.org/10.1016/j.bmc.2007.02.044] [PMID: 17349793]

[12] Kumar, A.; Sharma, S.; Archana, A.; Bajaj, K.; Sharma, S.; Panwar, H.; Singh, T.; Srivastava, V.K. Some new 2,3,6trisubstituted quinazolinones as potent anti-inflammatory, analgesic and COX-II inhibitors. Bioorg. Med. Chem., 2003, 11(23), 5293-5299.

[http://dx.doi.org/10.1016/S0968-0896(03)00501-7] [PMID: 14604693]

[13] Sharma, S.; Singh, T.; Mittal, R.; Saxena, K.K.; Srivastava, V.K.; Kumar, A. A study of anti-inflammatory activity of some novel I -amino naphthalene and I -amino naphthalene derivatives. Arch. Pharm. (Weinheim), 2006, 339(3), 145-152. [http://dx.doi.org/10.1002/ardp.200500215] [PMID: 16528795]

[14] Petrikaite, V.; Tarasevicius, E.; Pavilonis, A. New ethacridine derivatives as the potential antifungal and antibacterial preparations. Medicina (Kaunas), 2007, 43(8), 657-663. [PMID: 17895642]

[15] Chandrappa, S.; Kavitha, C.V.; Shahabuddin, M.S.; Vinaya, K.; Ananda Kumar, C.S.; Ranganatha, S.R.; Raghavan, S.C.; Rangappa, K.S. Synthesis of 2-(5-((5-(4-chlorophenyl)furan-2yl) methylene)-4-oxo-2-thioxothiazolidin-3-yl)acetic acid derivatives and evaluation of their cytotoxicity and induction of apoptosis in human leukemia cells. Bioorg. Med. Chem., 2009, 17(6), 2576-2584.

[http://dx.doi.org/10.1016/j.bmc.2009.01.016] [PMID: 19243955] 
[16] Murugan, R.; Anbazhagan, S.; Lingeshwaran, S.; Sriman Narayanan, S. Synthesis and in vivo antidiabetic activity of novel dispiropyrrolidines through $[3+2]$ cycloaddition reactions with thiazolidinedione and rhodanine derivatives. Eur. J. Med. Chem., 2009, 44(8), 3272-3279.

[http://dx.doi.org/10.1016/j.ejmech.2009.03.035] [PMID: 19395129]

[17] Brooke, E.W.; Davies, S.G.; Mulvaney, A.W.; Okada, M.; Pompeo, F.; Sim, E.; Vickers, R.J.; Westwood, I.M. Synthesis and in vitro evaluation of novel small molecule inhibitors of bacterial arylamine $\mathrm{N}$-acetyltransferases (NATs). Bioorg. Med. Chem. Lett., 2003, 13(15), 2527-2530.

[http://dx.doi.org/10.1016/S0960-894X(03)00484-0] [PMID: 12852958]

[18] Mallikarjuna, B.P.; Sastry, B.S.; Suresh Kumar, G.V.; Rajendraprasad, Y.; Chandrashekar, S.M.; Sathisha, K. Synthesis of new 4-isopropylthiazole hydrazide analogs and some derived clubbed triazole, oxadiazole ring systems--a novel class of potential antibacterial, antifungal and antitubercular agents. Eur. J. Med. Chem., 2009, 44(11), 4739-4746.

[http://dx.doi.org/10.1016/j.ejmech.2009.06.008] [PMID: 19589626]

[19] Hugel, H.M. Microwave multicomponent synthesis. Molecules, 2009, 14(12), 4936-4972.

[http://dx.doi.org/10.3390/molecules14124936] [PMID: 20032870]

[20] (a) Bazureau, J.P.; Mongin, F.; Hamelin, J.; Texier-Boullet, F. Microwave in Heterocyclic Chemistry. In: Microwaves Org. Synth, 2nd ed; Loupy, A., Eds.; Wiley-VCH: Weinheim, Germany, 2006; pp. 426-523. Chapter 10. [http://dx.doi.org/10.1002/9783527619559.ch10] ; (b) Besson, T.; Brain, C. Heterocyclic Chemistry Using Microwave Assisted Approaches. In: Microwaves Assisted Org. Synth; Tierney, J.P.; Lidström, P., Eds.; Blackwell Publishing, 2004. Chapter 3.

[21] Xu, L.L.; Zheng, C.J.; Sun, L.P.; Miao, J.; Piao, H.R. Synthesis of novel 1,3-diaryl pyrazole derivatives bearing rhodanine-3fatty acid moieties as potential antibacterial agents. Eur. J. Med. Chem., 2012, 48, 174-178.

[http://dx.doi.org/10.1016/j.ejmech.2011.12.011] [PMID: 22192483]

[22] Gualtieri, M.; Bastide, L.; Villain-Guillot, P.; MichauxCharachon, S.; Latouche, J.; Leonetti, J.P. In vitro activity of a new antibacterial rhodanine derivative against Staphylococcus epidermidis biofilms. J. Antimicrob. Chemother., 2006, 58(4), 778-783.

[http://dx.doi.org/10.1093/jac/dkl314] [PMID: 16880176]

[23] Sim, M.M.; Ng, S.B.; Buss, A.D.; Crasta, S.C.; Goh, K.L.; Lee, S.K. Benzylidene rhodanines as novel inhibitors of UDPN-acetylmuramate/L-alanine ligase. Bioorg. Med. Chem. Lett., 2002, 12(4), 697-699.

[http://dx.doi.org/10.1016/S0960-894X(01)00832-0] [PMID: 11844704]
[24] Sortino, M.; Delgado, P.; JuA rez, S.; Quiroga, J.; AbonA-a, R.; Insuasty, B.; Nogueras, M.; Rodero, L.; Garibotto, F.M.; Enriz, R.D.; Zacchino, S.A. Synthesis and antifungal activity of (Z)-5-arylidenerhodanines. Bioorg. Med. Chem., 2007, 15(1), 484-494.

[http://dx.doi.org/10.1016/j.bmc.2006.09.038]

[PMID: 17049255]

[25] Jin, X.; Zheng, C.J.; Song, M.X.; Wu, Y.; Sun, L.P.; Li, Y.J.; Yu, L.J.; Piao, H.R. Synthesis and antimicrobial evaluation of L-phenylalanine-derived C5-substituted rhodanine and chalcone derivatives containing thiobarbituric acid or 2thioxo-4-thiazolidinone. Eur. J. Med. Chem., 2012, 56, 203-209.

[http://dx.doi.org/10.1016/j.ejmech.2012.08.026] [PMID: 22982124]

[26] Chen, Z.H.; Zheng, C.J.; Sun, L.P.; Piao, H.R. Synthesis of new chalcone derivatives containing a rhodanine-3-acetic acid moiety with potential anti-bacterial activity. Eur. J. Med. Chem., 2010, 45(12), 5739-5743.

[http://dx.doi.org/10.1016/j.ejmech.2010.09.031] [PMID: 20889240]

[27] Zheng, C.J.; Song, M.X.; Sun, L.P.; Wu, Y.; Hong, L.; Piao, H.R. Synthesis and biological evaluation of 5-aryloxypyrazole derivatives bearing a rhodanine-3-aromatic acid as potential antimicrobial agents. Bioorg. Med. Chem. Lett., 2012, 22(23), 7024-7028.

[http://dx.doi.org/10.1016/j.bmcl.2012.09.107]

[PMID: 23099091]

[28] (a) Darandale, S.N.; Pansare, D.N.; Mulla, N.A.; Shinde, D.B. Green synthesis of tetrahydropyrimidine analogues and evaluation of their antimicrobial activity. Bioorg. Med. Chem. Lett., 2013, 23(9), 2632-2635.

[http://dx.doi.org/10.1016/j.bmcl.2013.02.099] [PMID: 23522562] ; (b) Darandale, S.N.; Mulla, N.A.; Pansare, D.N.; Sangshetti, J.N.; Shinde, D.B. A novel amalgamation of 1,2,3triazoles, piperidines and thieno pyridine rings and evaluation of their antifungal activity. Eur. J. Med. Chem., 2013, 65, 527-532.

[http://dx.doi.org/10.1016/j.ejmech.2013.04.045] [PMID: 23807083]

[29] (a) Pansare, D.N.; Shinde, D.B. A facile synthesis of (Z)-5-(substituted)-2-(methylthio)thiazol-4(5H)-one using microwave irradiation and conventional method. Tetrahedron Lett., 2014, 55, 1107-1110.

[http://dx.doi.org/10.1016/j.tetlet.2013.12.113] ; (b) Lesyk, R.B.; Zimenkovsky, B.S. 4-thiazolidones: centenarian history, current status and perspectives for modern organic and medicinal chemistry. Curr. Org. Chem., 2004, 8, 1547-1577. [http://dx.doi.org/10.2174/1385272043369773]

[30] Li, H.Q.; Yang, J.; Ma, S.; Qiao, C. Structure-based design of rhodanine-based acylsulfonamide derivatives as antagonists of the anti-apoptotic Bcl-2 protein. Bioorg. Med. Chem., 2012, 20(14), 4194-4200.

[http://dx.doi.org/10.1016/j.bmc.2012.05.079]

[PMID: 22739087]

(C) Pansare and Shinde; Licensee Bentham Open

This is an open access article licensed under the terms of the (https://creativecommons.org/licenses/by/4.0/legalcode), which permits unrestricted, noncommercial use, distribution and reproduction in any medium, provided the work is properly cited. 